\title{
Simulating ultrastrong-coupling processes breaking parity conservation in Jaynes-Cummings systems
}

\author{
Carlos Sánchez Muñoz $\odot,{ }^{1,2}$ Anton Frisk Kockum $\odot,{ }^{1,3}$ Adam Miranowicz $\odot,{ }^{1,4}$ and Franco Nori $\circledast^{1,5}$ \\ ${ }^{1}$ Theoretical Quantum Physics Laboratory, RIKEN Cluster for Pioneering Research, Wako-shi, Saitama 351-0198, Japan \\ ${ }^{2}$ Clarendon Laboratory, University of Oxford, Parks Road, Oxford OX1 3PU, United Kingdom \\ ${ }^{3}$ Wallenberg Centre for Quantum Technology, Department of Microtechnology and Nanoscience, \\ Chalmers University of Technology, 41296 Gothenburg, Sweden \\ ${ }^{4}$ Faculty of Physics, Adam Mickiewicz University, 61-614 Poznań, Poland \\ ${ }^{5}$ Department of Physics, The University of Michigan, Ann Arbor, Michigan 48109-1040, USA
}

(Received 7 November 2019; revised 10 August 2020; accepted 13 August 2020; published 11 September 2020)

\begin{abstract}
We propose the effective simulation of light-matter ultrastrong-coupling phenomena with strong-coupling systems. Recent theory and experiments have shown that the quantum Rabi Hamiltonian can be simulated by a Jaynes-Cummings system with the addition of two classical drives. This allows one to implement nonlinear processes that do not conserve the total number of excitations. However, parity is still a conserved quantity in the quantum Rabi Hamiltonian, which forbids a wide family of processes involving virtual transitions that break this conservation. Here, we show that these parity-nonconserving processes can be simulated and that this can be done in an even simpler setup: a Jaynes-Cummings-type system with the addition of a single classical drive. By shifting the paradigm from simulating a particular model to simulating a particular process, we are able to implement a much wider family of nonlinear coherent protocols than in previous simulation approaches, doing so with fewer resources and constraints. We focus our analysis on three particular examples: a single atom exciting two photons, frequency conversion, and a single photon exciting two atoms.
\end{abstract}

DOI: 10.1103/PhysRevA.102.033716

\section{INTRODUCTION}

The ultrastrong coupling (USC) of light and matter is attracting increasing interest beyond the fields of cavity and circuit quantum electrodynamics (QED) [1-5]. This interest has been stimulated in the last decade by several experiments finally reaching USC in a variety of physical systems [4,5]. The USC of light and matter (e.g., a cavity mode and a natural or artificial atom) occurs when their coupling strength $g$ becomes comparable to the atomic $\left(\omega_{a}\right)$ or cavity $\left(\omega_{c}\right)$ frequencies. More precisely, according to the usual convention, the USC regime occurs when $\eta=\max \left(g / \omega_{c}, g / \omega_{a}\right)$ is in the range $[0.1,1)$. The regime $\eta \geqslant 1$ is often referred to as deep strong coupling (DSC) [6].

Compared to strong coupling (SC; $\eta<0.1$, but $g$ larger than the loss rates in the system), USC opens new perspectives for efficiently simulating known effects and observing fundamentally new phenomena in quantum nonlinear optics [7-17], quantum field theory, supersymmetric (SUSY) field theories [18], cavity optomechanics [19-26], quantum plasmonics [21,27-29], light-induced superconductivity [30,31], quantum thermodynamics [32], photochemistry (chemistry QED) [33-36], as well as metamaterial and material sciences. Ultrastrong coupling also has applications in quantum metrology and spectroscopy [37], and quantum information processing [13,38-43].

The basic model for USC of a single two-level atom to a single-mode cavity is the quantum Rabi model $[44,45]$
(QRM). Its multiatom or multimode generalizations include the Dicke [46] and Hopfield [47] models. When $\eta<0.1$, these models for USC can be reduced to the simpler JaynesCummings model [48] (JCM) and its multimode or multiatom generalizations (e.g., the Tavis-Cummings model [49]). Since SC is typically easier to realize in experiment than USC, the question arises whether the predicted USC phenomena can be observed or at least simulated also in the SC regime, e.g., by adding classical drives applied to atom(s) or cavity mode(s) in the SC models. We note that simulating the QRM could also enable simulating other closely related fundamental quantum models, which include the spin-boson [50,51] and Kondo [50,52,53] renormalization-group models, the RashbaDresselhaus model [18], and the Jahn-Teller model [54-58], among others. Even vacuum-induced symmetry breaking [59], which is analogous to the Higgs mechanism, has been predicted in the USC regime. Quantum simulations of the atom-cavity dynamics in the USC and DSC regimes in the Rabi and Dicke models have recently attracted much theoretical [60-72] and experimental [73-76] interest. The methods described in Refs. [61,63] and implemented in circuit-QED [74] and trapped-ion [76] experiments simulate the QRM in the USC regime with a light-matter system described by the JCM in the SC regime. These quantum simulations require two drives to be applied to a system with a single atom and a single-mode resonator.

In this work, we propose to shift the paradigm from simulating the full QRM to simulating particular processes 
characteristic of the USC regime, e.g., violating the conservation of number of particles. This approach allows one to employ fewer resources and to go beyond the standard QRM and implement hallmark USC processes that are forbidden due to parity conservation [8,10-13]. We illustrate this approach by analyzing three different phenomena that require breaking parity conservation: in Sec. III, we study a single two-level atom emitting two photons [8]; in Sec. IV, frequency conversion of two photonic modes coupled to a two-level atom [12]; and in Sec. V, a single photon exciting two atoms [10]. In Sec. VI, we give a protocol for an experimental implementation and show its feasibility in several, well-developed experimental systems. Finally, in Sec. VII, we study the effect of decoherence.

\section{HAMILTONIANS FOR LIGHT-MATTER COUPLING}

The QRM describes the interaction between a two-level atom (qubit) of frequency $\omega_{a}$ and a cavity mode of frequency $\omega_{c}$ by the Hamiltonian $(\hbar=1)$,

$$
H_{R}=H_{0}+\sigma_{x} X=H_{0}+g\left(\sigma+\sigma^{\dagger}\right)\left(a+a^{\dagger}\right),
$$

where

$$
H_{0}=\frac{1}{2} \omega_{a} \sigma_{z}+\omega_{c} a^{\dagger} a
$$

is the free Hamiltonian, while $a\left(a^{\dagger}\right)$ is the annihilation (creation) operator of the cavity mode, $X=a+a^{\dagger}$ is the canonical position operator, $\sigma_{x}=\sigma+\sigma^{\dagger}$ and $\sigma_{z}$ are Pauli operators, $\sigma\left(\sigma^{\dagger}\right)$ is the atomic lowering (raising) operator, and $g$ is the atom-field coupling constant. Under the rotatingwave approximation (RWA), which is valid if $\left\{\omega_{c}, \omega_{a}\right\} \gg$ $\left\{g,\left|\omega_{c}-\omega_{a}\right|\right\}$, the counter-rotating terms, $\sigma^{\dagger} a^{\dagger}$ and $\sigma a$, in Eq. (1) can be ignored. This leads to the standard JCM described by the Hamiltonian

$$
H_{\mathrm{JC}}=H_{0}+g\left(\sigma a^{\dagger}+\sigma^{\dagger} a\right)
$$

The counter-rotating terms can be effectively restored in the JCM in various ways, e.g., using cavity-light squeezing $[69,70]$ to enhance the coupling strength $g$. A simpler method is to apply two time-dependent classical drives, as suggested in Ref. [61]. This yields an effective QRM Hamiltonian in a rotated frame, where the ratio $\eta \equiv g / \omega_{c}$ can be effectively increased as

$$
\eta^{\prime} \equiv \frac{g^{\prime}}{\omega_{c}^{\prime}}=\frac{g}{2\left(\omega_{c}-\omega_{1}\right)}
$$

(with $\omega_{1}$ one of the driving frequencies) from the SC regime up to the USC regime, or even the DSC regime. In this scheme, the effective frequency of the qubit is equal to the amplitude of one of the drives.

Equation (1), however, does not include any term that changes the number of particles by an odd number, and thus parity is conserved. We now show how a simpler JCM setup, with only a single drive, can be used to simulate any particular process characteristic of the USC, i.e., relying on counter-rotating terms in the QRM, but also violating parity conservation through terms of the type $\sigma_{z}\left(a+a^{\dagger}\right)$. Our approach is inspired by earlier work on creating multiphoton states in cavity QED [77-79].
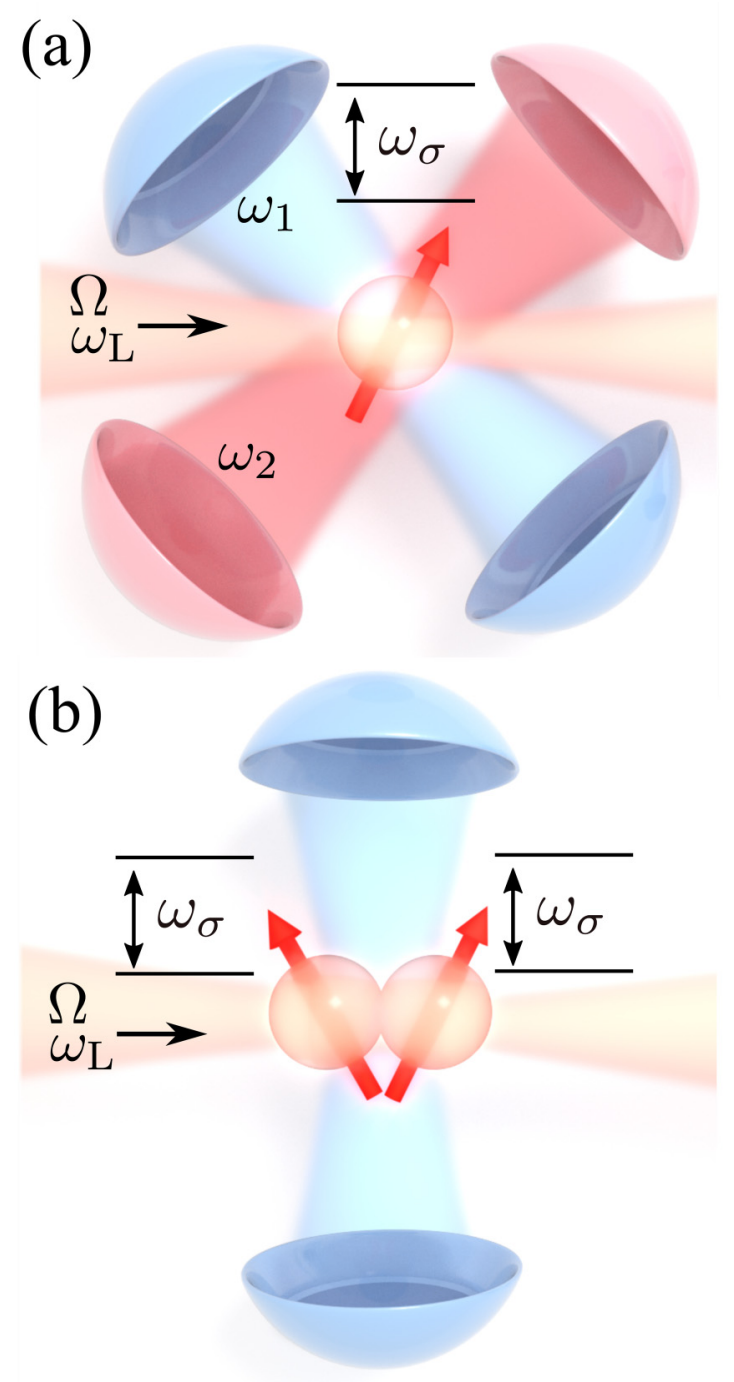

FIG. 1. Sketches of the two setups that we consider for observing ultrastrong-coupling phenomena. (a) A single two-level atom of frequency $\omega_{\sigma}$ coupled to two cavities of frequencies $\omega_{1}$ and $\omega_{2}$. (b) Two two-level atoms of frequency $\omega_{\sigma}$ coupled to a cavity of frequency $\omega_{a}$. In both setups, a single coherent drive of frequency $\omega_{\mathrm{L}}$ and amplitude $\Omega$ is applied to each atom.

\section{USC PROCESS I: TWO PHOTONS EXCITED BY A SINGLE ATOM}

We first consider the setup in Fig. 1(a), i.e., two cavities coupled to a single qubit that is coherently driven by a classical field. In a frame rotating with the frequency $\omega_{\mathrm{L}}$ of the driving field, the Hamiltonian is given by

$$
\begin{aligned}
H= & \Delta_{1} a_{1}^{\dagger} a_{1}+\Delta_{2} a_{2}^{\dagger} a_{2}+\Delta_{\sigma} \sigma^{\dagger} \sigma+\Omega\left(\sigma+\sigma^{\dagger}\right) \\
& +g\left[\sigma\left(a_{1}^{\dagger}+a_{2}^{\dagger}\right)+\text { H.c. }\right],
\end{aligned}
$$

with $a_{1,2}$ the bosonic annihilation operators of the cavity modes; $\Delta_{1}, \Delta_{2}$, and $\Delta_{\sigma}$ are the frequency detunings between the cavities or qubit and the drive $\left(\Delta_{x} \equiv \omega_{x}-\omega_{\mathrm{L}}\right), \Omega$ is the amplitude of the driving field, and $g$ is the coupling rate between the cavities and the qubit (considered to be equal, for simplicity). The part of Eq. (5) that only depends on $\sigma$ can be 

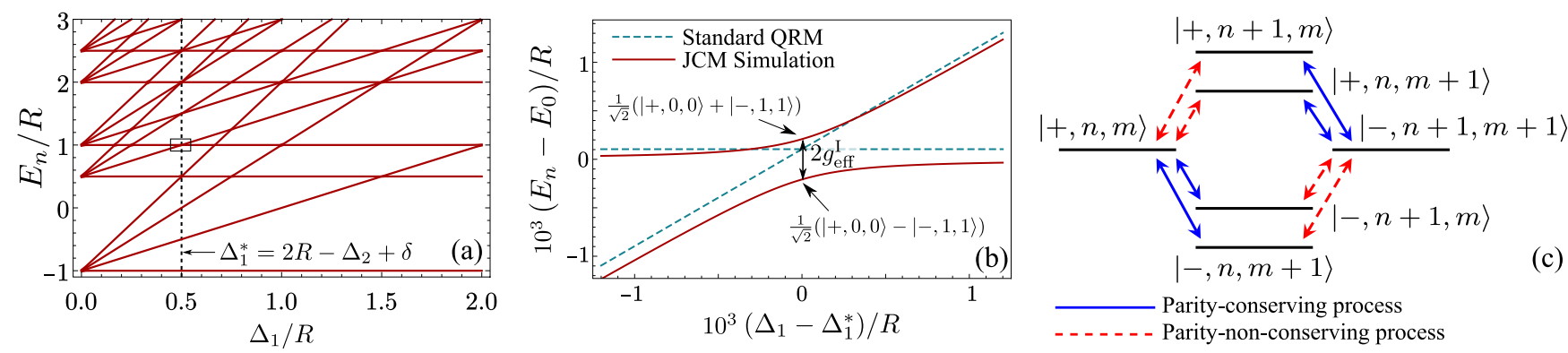

FIG. 2. USC process I: Energy-level diagrams and transitions for the process where a single atom emits two photons. (a) Energy levels $E_{n}$ for the Hamiltonian in Eq. (11) as a function of $\Delta_{1}$. Parameters: $\Omega=80 g, \Delta_{\sigma}=\Omega / \sqrt{2}, \Delta_{2}=3 R / 2$, with $R=\sqrt{\Omega^{2}+\Delta_{\sigma}^{2} / 4}$. The Hilbert space is truncated at three photons for simplicity. (b) Zoom-in on the anticrossing between the energy levels corresponding to $|+, 0,0\rangle$ and $|-, 1,1\rangle$. The size of the level splitting at the resonance $\Delta_{1}=\Delta_{1}^{*} \approx 2 R-\Delta_{2}-$ where both states have the same energy in the absence of coupling $E_{0}=\Delta_{2}-R=R$-indicates the strength $g_{\text {eff }}^{\mathrm{I}}$ of the effective interaction between these two states. (c) The transitions in the second-order process that creates the effective coupling between $|+, n, m\rangle$ and $|-, n+1, m+1\rangle$. Red dashed (blue solid) arrows indicate transitions that change the total number of excitations in the system by one (zero), i.e., transitions mediated by counter-rotating (nonrotating) terms. Crucially, the whole process cannot occur without involving intermediate, parity-nonconserving processes.

easily diagonalized. Denoting the ground and excited eigenstates of an undriven qubit $|g\rangle$ and $|e\rangle$, respectively, the new eigenstates with the driving applied correspond to a rotated spin basis, i.e.,

$$
\begin{gathered}
|+\rangle=\cos \theta|g\rangle+\sin \theta|e\rangle=e^{i \sigma_{y} 2 \theta}|g\rangle, \\
|-\rangle=\sin \theta|g\rangle-\cos \theta|e\rangle=-e^{i \sigma_{y}(2 \theta+\pi)}|g\rangle,
\end{gathered}
$$

with $\cos \theta \equiv 1 / \sqrt{1+\xi^{-2}}, \sin \theta \equiv 1 / \sqrt{1+\xi^{2}}, \theta \in[0, \pi / 2]$, and $\xi \equiv \Omega /\left(\Delta_{\sigma} / 2+R\right)$, where $R$ is the Rabi frequency given by

$$
R \equiv \sqrt{\Omega^{2}+\left(\Delta_{\sigma} / 2\right)^{2}} .
$$

Working in the eigenbasis $| \pm\rangle$, the original lowering operator $\sigma$ can be written in terms of the new operators,

$$
\tilde{\sigma} \equiv|-\rangle+\mid, \quad \tilde{\sigma}_{z} \equiv 2 \tilde{\sigma}^{\dagger} \tilde{\sigma}-\mathbb{1},
$$

since

$$
\sigma=s^{2} \tilde{\sigma}-c^{2} \tilde{\sigma}^{\dagger}+c s \tilde{\sigma}_{z},
$$

with $s=\sin \theta$ and $c=\cos \theta$. Therefore, the resulting Hamiltonian in the rotated spin basis reads

$$
\begin{aligned}
H= & \Delta_{1} a_{1}^{\dagger} a_{1}+\Delta_{2} a_{2}^{\dagger} a_{2}+R \tilde{\sigma}_{z} \\
& +g\left[\left(s^{2} \tilde{\sigma}-c^{2} \tilde{\sigma}^{\dagger}+c s \tilde{\sigma}_{z}\right)\left(a_{1}^{\dagger}+a_{2}^{\dagger}\right)+\text { H.c. }\right] .
\end{aligned}
$$

The transition energy of the effective qubit is now given by $R$, which can be made small enough that counter-rotating terms of the kind $\tilde{\sigma}^{\dagger} a_{1}^{\dagger}$ and $\tilde{\sigma}_{z} a_{1}^{\dagger}$ play a relevant role in the dynamics. The presence of the latter type of coupling terms, involving $\tilde{\sigma}_{z}$, makes $H$ reminiscent of the generalized QRM, where a coupling term proportional to $\sigma_{z}\left(a+a^{\dagger}\right)$ is added to the QRM in Eq. (1) $[7,8,10,11,80,81]$. Crucially, the presence of the $\tilde{\sigma}_{z}$ coupling term, which was absent in previous proposals of simulation of the QRM, breaks parity symmetry and enables processes that change the number of excitations in the system by an odd number [4,5,11].

We will now see how, in the limit of $\alpha \equiv g / R \ll 1$, the counter-rotating terms in Eq. (11) lead to Rabi oscillations between pairs of eigenstates of the bare Hamiltonian that are not directly coupled by the interactions [11], with Rabi frequencies $\propto \alpha g$. In the effective USC regime when $\alpha \sim 0.1$, we find the optimal condition in which $g / R \ll 1$ remains valid, while the effective Rabi frequencies $\sim 0.1 \mathrm{~g}$ can be significant compared to decoherence rates. The normal USC condition $\eta \gtrsim 0.1$ for observing these phenomena is thus lifted.

One example of such a nonlinear process is the simultaneous excitation of one photon in each cavity by the single qubit. By plotting the energy levels of Eq. (11) [Fig. 2(a)] and zooming in around $\Delta_{1}+\Delta_{2} \approx 2 R$, we find an avoidedlevel crossing [Fig. 2(b)]. The interaction around this point is described by the effective Hamiltonian (see Appendix A)

$$
\begin{aligned}
H_{\mathrm{eff}}^{\mathrm{I}}= & \Delta_{1} a_{1}^{\dagger} a_{1}+\Delta_{2} a_{2}^{\dagger} a_{2}+(R+\lambda) \tilde{\sigma}_{z} \\
& +\left(\chi_{1} a_{1}^{\dagger} a_{1}+\chi_{2} a_{2}^{\dagger} a_{2}\right) \tilde{\sigma}_{z}+g_{\mathrm{eff}}^{\mathrm{I}}\left(a_{1}^{\dagger} a_{2}^{\dagger} \tilde{\sigma}+\text { H.c. }\right),
\end{aligned}
$$

which couples the states $|+, n, m\rangle \leftrightarrow|-, n+1, m+1\rangle$, confining the dynamics inside that manifold. This effective interaction requires both states to be quasiresonant, which implies, ignoring for now small dispersive energy shifts, the two conditions

$$
\Delta_{1}+\Delta_{2} \approx 2 R, \quad \Delta_{1} \neq \Delta_{2} \neq( \pm R, \pm 2 R) .
$$

The second condition is imposed in order to be detuned from first-order processes (e.g., $\tilde{\sigma} a_{1}^{\dagger}+$ H.c. if $\Delta_{1}=2 R$ ) and competing second-order processes [e.g., $\left(\tilde{\sigma} a_{1}^{\dagger}+\right.$ H.c.) for $\Delta_{1}=$ $R]$ exciting degenerate photon pairs within a single cavity [77-79]. The effective two-photon coupling rate in Eq. (12) is given by

$$
g_{\text {eff }}^{\mathrm{I}}=g^{2} c s^{3}[R f(1-f)]^{-1},
$$

where we defined

$$
\Delta_{1}=2 f R, \quad \Delta_{2}=(1-f) 2 R
$$

for $f \in(0,1)$, so that Eq. (16) is automatically fulfilled. This effective interaction is mediated by the second-order processes shown in Fig. 2(c). The Lamb shift of the qubit is

$$
\lambda=g^{2}\left[c^{4}\left(\Delta_{1,+}^{-1}+\Delta_{2,+}^{-1}\right)-s^{4}\left(\Delta_{1,-}^{-1}+\Delta_{2,-}^{-1}\right)\right] / 2,
$$



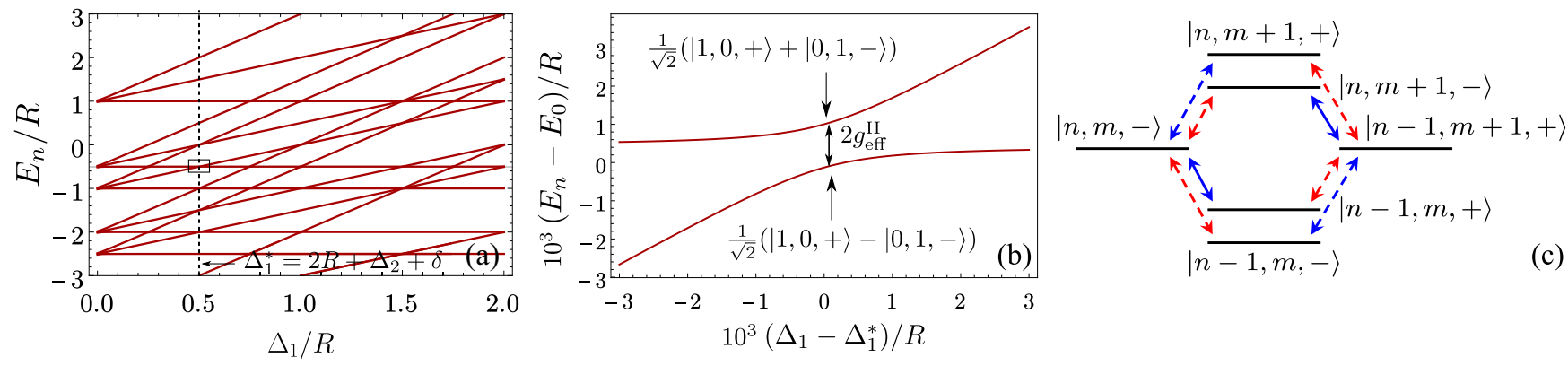

FIG. 3. USC effect II: Energy-level diagrams and transitions for the process of frequency conversion. (a) Energy levels $E_{n}$ for the Hamiltonian in Eq. (11) as a function of $\Delta_{1}$. Parameters: $\Omega=40 g, \Delta_{\sigma}=\Delta_{\sigma}^{*}$ [see Eq. (C9)], $\Delta_{2}=2 R(f-1)$, with $f=1 / 4$ and $R=\sqrt{\Omega^{2}+\Delta_{\sigma}^{2} / 4}$. The Hilbert space is truncated at 1 photon for simplicity. (b) Zoom-in on the anticrossing between the energy levels corresponding to $|1,0,+\rangle$ and $|0,1,-\rangle$. The size of the level splitting at the resonance $\Delta_{1}=\Delta_{1}^{*}=2 R+\Delta_{2}+\delta=2 R f+\delta$-where both states have the same energy in the absence of coupling $E_{0}=\Delta_{2}+R=R(2 f-1)$-indicates the strength $g_{\text {eff }}^{\text {II }}$ of the effective interaction between these two states. (c) The transitions in the second-order process that creates the effective coupling between $|n, m,-\rangle$ and $|n-1, m+1,+\rangle$. Blue solid arrows: transitions that conserve the number of excitations. Red dashed arrows: transitions that change the number of excitations by one. Blue dashed arrows: transitions that change the number of excitations by two.

and the dispersive coupling rates are

$$
\chi_{i}=g^{2}\left(\frac{c^{4}}{\Delta_{i,+}}-\frac{s^{4}}{\Delta_{i,-}}\right),
$$

with $\Delta_{i, \pm} \equiv \Delta_{i} \pm 2 R$. Equation (17) shows that the resonantdriving condition $\Delta_{\sigma}=0(\theta=\pi / 4)$ does not provide the maximum possible two-photon coupling rate. In particular, for a fixed $R$, we see that the optimal angle $\theta$ maximizing $g_{\text {eff }}^{\mathrm{I}}$ is $\theta^{*}=\pi / 3$. This angle yields the optimum value $g_{\text {eff }}^{\mathrm{I}}\left(\theta^{*}\right) \approx$ $1.3 g_{\text {eff }}^{\mathrm{I}}(\theta=\pi / 4)$. Alternatively, we can compute the optimal detuning $\Delta_{\sigma}$ for a fixed $\Omega$, which is experimentally more meaningful since varying $\Delta_{\sigma}$ for a fixed $\Omega$ is more straightforward than varying $\theta$ for a fixed $R$. By writing Eq. (17) explicitly in terms of $\Delta_{\sigma}$ and $\Omega$, we obtain the optimal detuning $\Delta_{\sigma}^{*}=\Omega / \sqrt{2}$. The corresponding value of $g_{\text {eff }}^{\mathrm{I}}$ is then given by $g_{\text {eff }}^{\mathrm{I}}\left(\Delta_{\sigma}^{*}\right) \approx 1.18 g_{\text {eff }}^{\mathrm{I}}\left(\Delta_{\sigma}=0\right)$.

In order to obtain full two-photon Rabi oscillations between the states $|1\rangle=|+, n, m\rangle$ and $|2\rangle=|-, n+1, m+1\rangle$, the quasiresonance condition given by Eq. (16) needs to be fine-tuned to account for the Lamb shift of the qubit and the dispersive qubit-cavity couplings in Eq. (12), given by $\lambda$ and $\chi_{1}, \chi_{2}$. In other words, $\Delta_{1}$ and $\Delta_{2}$ must be chosen such that $\left\langle 1 \mid H_{\text {eff }}\right\rangle 1=\left\langle 2 \mid H_{\text {eff }}\right\rangle 2$. Introducing a correction $\delta$ such that $\Delta_{1}=2 R f+\delta$, we solve this equation for the Hamiltonian in Eq. (12) and obtain

$$
\delta=2 \lambda+\chi_{1}(2 n+1)+\chi_{2}(2 m+1) .
$$

More details about the procedure outlined above can be found in Appendix B.

\section{USC PROCESS II: FREQUENCY CONVERSION}

The setup in Fig. 1(a) can also be exploited to engineer other processes, e.g., frequency conversion. In that case, we want to couple the states $|n+1, m,-\rangle$ and $|n, m+1,+\rangle$, see Fig. 3. The resonance conditions then become

$$
\Delta_{1} \approx 2 R+\Delta_{2}, \quad \Delta_{1} \neq \Delta_{2} \neq \pm R
$$

where, again, the second condition guarantees that secondorder processes introducing photon pairs into the cavities are off-resonance. Following the same procedure outlined in Appendix $\mathrm{C}$, we obtain the effective Hamiltonian

$$
\begin{aligned}
H_{\mathrm{eff}}^{\mathrm{II}}= & \Delta_{1} a_{1}^{\dagger} a_{1}+\Delta_{2} a_{2}^{\dagger} a_{2}+(R+\lambda) \tilde{\sigma}_{z} \\
& +\left(\chi_{1} a_{1}^{\dagger} a_{1}+\chi_{2} a_{2}^{\dagger} a_{2}\right) \tilde{\sigma}_{z}+g_{\mathrm{eff}}^{\mathrm{II}}\left(a_{1}^{\dagger} a_{2} \tilde{\sigma}+\text { H.c. }\right)
\end{aligned}
$$

where the frequency-conversion rate is given by

$$
g_{\mathrm{eff}}^{\mathrm{II}}=g^{2} \frac{(f-1) c^{3} s+f c s^{3}}{R f(f-1)},
$$

having now defined

$$
\Delta_{1}=2 f R, \quad \Delta_{2}=(f-1) 2 R
$$

for $f \in(0,1)$. Once again, driving the qubit on resonance does not maximize $g_{\text {eff }}^{\mathrm{II}}$. Frequency-conversion-rate increases of $50-70 \%$ compared to resonant driving can be achieved by using the optimal angle $\theta^{*}$ or the optimal detuning $\Delta^{*}$, whose analytical expressions can be found in Appendix C.

\section{USC PROCESS III: TWO ATOMS EXCITED BY A SINGLE PHOTON}

The last process that we demonstrate is the excitation of two atoms by a single photon, i.e., the direct coupling between the states $|+,+, n\rangle$ and $|-,-, n+1\rangle$, see Fig. 4. We now consider the setup in Fig. 1(b), i.e., a cavity coupled to two coherently driven qubits, with lowering operators $\sigma_{1,2}$. For simplicity and without loss of generality, we consider both qubits to have the same transition frequencies. In the rotating frame of the driving, the Hamiltonian is

$$
\begin{aligned}
H= & \Delta_{a} a^{\dagger} a+\Delta_{\sigma}\left(\sigma_{1}^{\dagger} \sigma_{1}+\sigma_{2}^{\dagger} \sigma_{2}\right)+\Omega\left(\sigma_{1}+\sigma_{2}+\text { H.c. }\right) \\
& +g\left[a\left(\sigma_{1}^{\dagger}+\sigma_{2}^{\dagger}\right)+\text { H.c. }\right],
\end{aligned}
$$

where $a$ is the bosonic annihilation operator of the cavity, and $\Delta_{a}=\omega_{a}-\omega_{\mathrm{L}}\left(\Delta_{\sigma}=\omega_{\sigma}-\omega_{\mathrm{L}}\right)$ is the cavity (qubit) detuning from the drive frequency. In the dressed-qubits basis, the resonance condition enabling the desired nonlinear process simply 
(a)

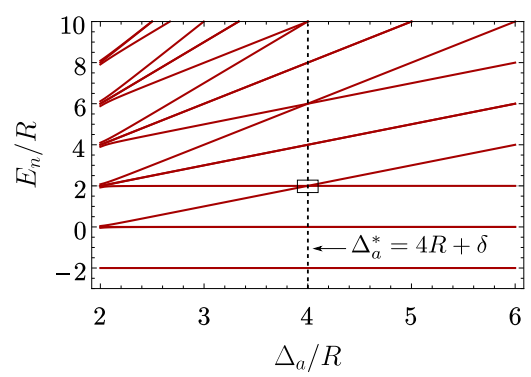

(b)

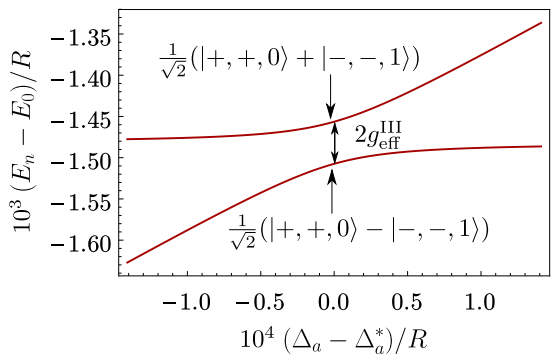

(c)

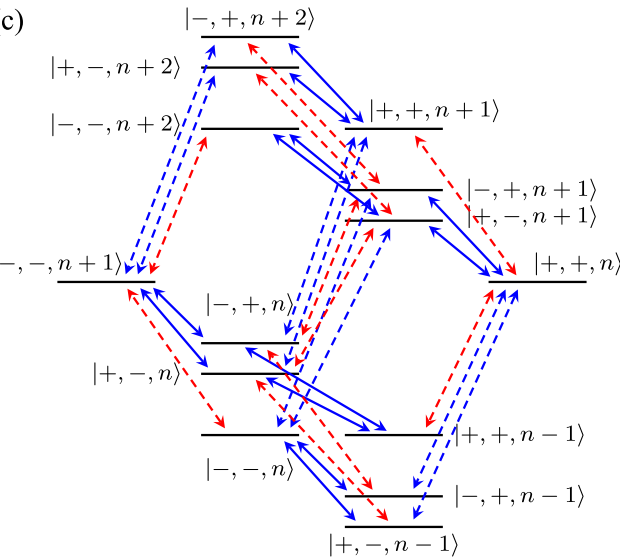

FIG. 4. USC effect III: Energy-level diagrams and transitions for the process of exciting two atoms with a single photon. (a) Energy levels $E_{n}$ for the Hamiltonian in Eq. (23) as a function of $\Delta_{a}$. Parameters: $\Omega=20 g, \Delta_{\sigma}=\Delta_{\sigma}^{*}$ [see Eq. (D6)], with $R=\sqrt{\Omega^{2}+\Delta_{\sigma}^{2} / 4}$. The Hilbert space is truncated at four photons, for simplicity. (b) Zoom-in on the anticrossing between the energy levels corresponding to $|+,+, 0\rangle$ and $|-,-, 1\rangle$. The size of the level splitting at the resonance $\Delta_{a}=\Delta_{a}^{*}=4 R+\delta$-where both states have the same energy in the absence of coupling $E_{0}=2 R$-indicates the strength $g_{\text {eff }}^{\text {III }}$ of the effective interaction between these two states. (c) The transitions in the third-order process that creates the effective coupling between $|-,-, n+1\rangle$ and $|+,+, n-1\rangle$. Blue solid arrows: transitions that conserve the number of excitations. Red dashed arrows: transitions that change the number of excitations by one. Blue dashed arrows: transitions that change the number of excitations by two.

reads $\Delta_{a} \approx 4 R$. We then obtain (see Appendix D for details) the effective Hamiltonian

$$
\begin{aligned}
H_{\mathrm{eff}}^{\mathrm{III}}= & \Delta_{a} a^{\dagger} a+\sum_{i}(R+\lambda) \tilde{\sigma}_{z, i}+\chi a^{\dagger} a \tilde{\sigma}_{z, i} \\
& +g_{\mathrm{eff}}^{\mathrm{III}}\left(a^{\dagger} \sigma_{1} \sigma_{2}+a \sigma_{1}^{\dagger} \sigma_{2}^{\dagger}\right),
\end{aligned}
$$

with an effective coupling rate that emerges from third-order processes,

$$
g_{\text {eff }}^{\text {III }}=\frac{g^{3}}{3 R^{2}}\left(c^{3} s^{3}+3 c s^{5}\right) .
$$

As in the previous cases, the effective coupling can be maximized by driving the qubit slightly off resonance, using either the optimal angle $\theta^{*}$ or the optimal detuning $\Delta^{*}$, whose expressions we provide in Appendix D. The validity of our perturbative methods applied in Secs. III-V has been numerically tested in Appendix E. There it is shown that the exact result matches perturbation theory providing that the driving amplitude fulfills $\Omega \geqslant 10 \mathrm{~g}$.

\section{EXPERIMENTAL IMPLEMENTATIONS}

\section{A. Effective nonlinear coupling rates for realistic setups}

The results presented here are based on very fundamental models that describe the exchange of single excitations between a qubit and a harmonic oscillator, and can, therefore, be applied in many different systems. In Table I, we compare, under experimentally feasible assumptions, the effective coupling strengths $g_{\text {eff }}$ and decoherence rates $\gamma$ that can be obtained in several experimental platforms. An experimental implementation is feasible when $g_{\text {eff }} / \gamma>1$, i.e., when the effective coupling is strong. Table I shows that the secondorder processes that we have proposed here should be ready for implementation in several systems, and the third-order process may be within reach for circuit-QED setups. However, we note that the nonlinear processes may also be exploited even in the dissipative regime where $g_{\text {eff }}<\gamma$, for instance yielding multiphoton emission with nonclassical properties [77-79,87].

\section{B. Experimental protocol for observing nonlinear processes}

We now discuss an experimental protocol for implementing and measuring the nonlinear processes presented above, focusing without loss of generality on the USC process I, introduced in Sec. III. This protocol is shown in Fig. 5. Starting with no photons in the cavities and the qubit in its ground state $|g\rangle$, the first step is to apply a rotation of $2 \theta$ around the $y$ axis to bring the qubit into the eigenstate $|+\rangle$. This single-qubit rotation is a basic element of any quantum information toolbox. At this stage, the cavities and the qubit are detuned and no interaction takes place. Then, the driving field is switched on and the nonlinear process becomes resonant. After the system has evolved for a time $t$, the drive is switched off (effectively decoupling the qubit and the cavity), and the state of the qubit

TABLE I. Experimentally feasible effective rates for the three processes discussed in the text: (I) a single photon exciting two atoms, (II) frequency conversion, and (III) a single atom exciting two photons. We set $\Omega / g=10 ; \gamma$ refers to the largest decoherence rate in the system.

\begin{tabular}{lccc}
\hline \hline System & $g /(2 \pi)$ & $\gamma /(2 \pi)$ & $\left(g_{\text {eff }}^{\text {I }}, g_{\text {eff }}^{\text {II }}, g_{\text {eff }}^{\text {III }}\right) / \gamma$ \\
\hline Natural atoms [82] & $34 \mathrm{MHz}$ & $4.1 \mathrm{MHz}$ & $(1.2,0.8,0.02)$ \\
Trapped ions [83] & $10 \mathrm{kHz}$ & $100 \mathrm{~Hz}$ & $(15.8,10.1,0.22)$ \\
Quantum acoustics [84] & $16 \mathrm{MHz}$ & $0.6 \mathrm{MHz}$ & $(4.2,2.7,0.06)$ \\
Circuit QED [85] & $57 \mathrm{MHz}$ & $0.4 \mathrm{MHz}$ & $(22.5,14.4,0.3)$ \\
Quantum dots [86] & $19.3 \mathrm{GHz}$ & $6.0 \mathrm{GHz}$ & $(0.5,0.3,0.007)$ \\
\hline \hline
\end{tabular}


(a)

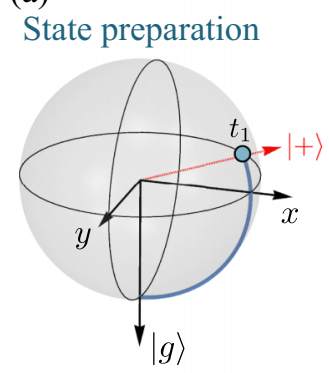

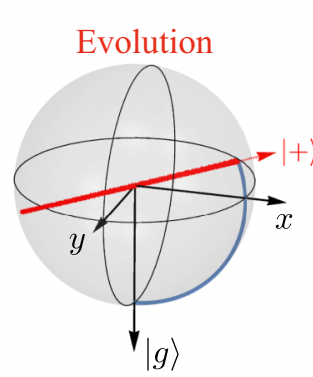

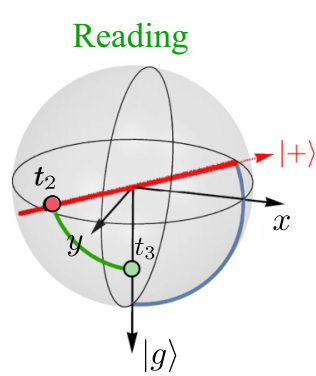

(b)

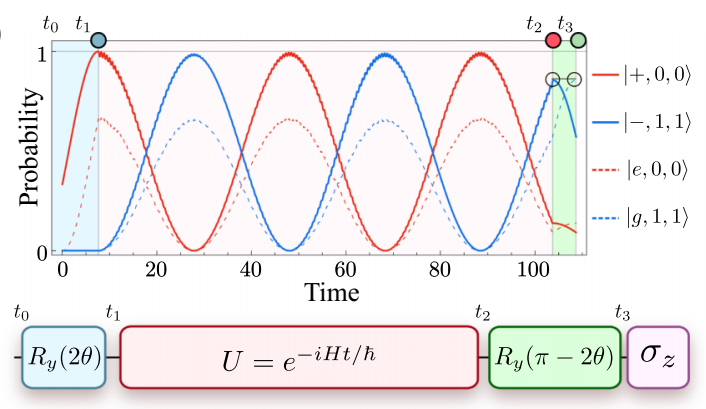

FIG. 5. USC process I: Illustration of the experimental protocol, showing (a) the Bloch sphere picture of the qubit state, (b) the time evolution of the occupation probabilities for the most relevant states, and (c) a schematic diagram of the proposed pulse sequence. From time $t_{0}$ to $t_{1}$, the qubit is rotated to the correct initial state in the rotated spin basis. From time $t_{1}$ to $t_{2}$, the qubit is driven and the system evolves according to the Hamiltonian in Eq. (11), i.e., moving back and forth along the red trajectory depicted in the Bloch sphere. At time $t_{2}$, the drive is turned off and the qubit is rotated back to the original basis, where it is then measured at time $t_{3}$.

in the $| \pm\rangle$ basis is transformed back into the $\{|g\rangle,|e\rangle\}$ basis (eigenstates of $\sigma_{z}$ ) by applying a rotation of $(\pi-2 \theta)$ around the $y$ axis. A measurement of the qubit population in the $\{|g\rangle,|e\rangle\}$ basis then reveals the qubit final state in the rotated basis. Repeating this process with different evolution times allows to reconstruct the multiphoton Rabi oscillations.

\section{EFFECT OF DECOHERENCE}

Here we provide further study of the effect of decoherence (beyond Table I), computing the dynamics of the nonlinear processes in the presence of cavity losses. This is done by using the standard Lindblad master equation for the dynamics of the density matrix,

$$
\dot{\rho}=-i[H, \rho]+\frac{\gamma_{a}}{2} \sum_{i=1}^{N_{\text {cav }}}\left(2 a_{i} \rho a_{i}-a_{i}^{\dagger} a_{i} \rho-\rho a_{i}^{\dagger} a_{i}\right),
$$

where $\gamma_{a}$ is a cavity decay rate, and the sum runs over the total number of cavities (two in cases I and II; one in case III). In Fig. 6, we show that the effect of decoherence is the expected damping of the Rabi oscillations. The apparent higher robustness to losses of case III (two atoms excited by a single photon) can be explained by the fact that there is only one cavity, which is the only lossy subsystem, as compared to two cavities in cases I and II. We have assumed the cavity decay to be the main decoherence mechanism, therefore ignoring the decay or dephasing of the atoms to simplify the discussion.

\section{CONCLUSIONS}

We have shown how, rather than simulating the full QRM with a JCM, one can simulate specific nonlinear processes characteristic of the USC regime. This requires fewer resources and allows one to implement processes that are forbidden in the standard QRM due to parity conservation. Our method is ready for its implementation on several existing experimental platforms, including cases, such as cavity QED with few emitters in the optical regime, where the USC is currently out of reach due to the high natural frequencies involved. This opens up new possibilities for exploring USC physics and its applications in technologies such as quantum information and quantum metrology.
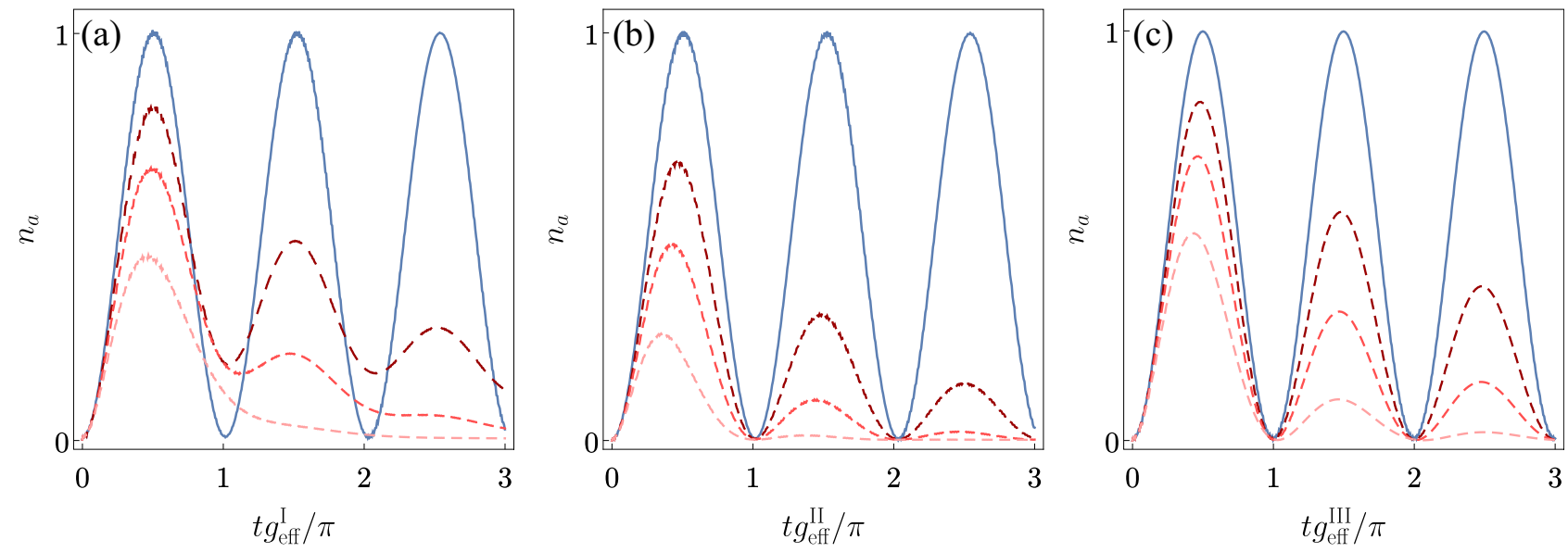

FIG. 6. Effect of decoherence induced by radiative decay in the cavity modes for the three nonlinear processes considered in this work. In each case, we monitor the population $n_{a}=\left\langle a^{\dagger} a\right\rangle$ of a cavity undergoing Rabi oscillations under the nonlinear processes with and without decay. Lossless dynamics are represented by straight, blue curves. Dissipative dynamics are shown by dashed curves, with lighter color representing higher decay rates. The decay rates used are $g_{\text {eff }} \times(0.25,0.5,1)$. (a) $\Omega / g=20$, (b) 20 , and (c) 10 . 


\section{ACKNOWLEDGMENTS}

C.S.M. is funded by the Marie Sklodowska-Curie Fellowship QUSON (Project No. 752180). A.F.K. acknowledges support from the Japan Society for the Promotion of Science (BRIDGE Fellowship BR190501), the Swedish Research Council (Grant No. 2019-03696), and the Knut and Alice Wallenberg Foundation through the Wallenberg Centre for Quantum Technology (WACQT). A.M. was supported by the Polish National Science Centre (NCN) under the Maestro Grant No. DEC-2019/34/A/ST2/00081. F.N. is supported in part by NTT Research, Army Research Office (ARO) (Grant No. W911NF-18-1-0358), Japan Science and Technology Agency (JST) (via Q-LEAP and the CREST Grant No. JPMJCR1676), Japan Society for the Promotion of Science (JSPS) (via the KAKENHI Grant No. JP20H00134, and the JSPS-RFBR Grant No. JPJSBP120194828), and Grant No. FQXi-IAF19-06 from the Foundational Questions Institute Fund (FQXi), a donor advised fund of the Silicon Valley Community Foundation. The project that gave rise to these results received the support of a fellowship from "la Caixa" Foundation (ID 100010434) and from the European Union's Horizon 2020 Research and Innovation Programme under the Marie Skłodowska-Curie Grant Agreement No. 47648. The fellowship code is LCF/BQ/PI20/11760026.

\section{APPENDIX A: EFFECTIVE HAMILTONIANS}

In this work, we use a matrix form of perturbation theory that allows one to obtain energy corrections to arbitrary orders with a single matrix inversion. Let us consider a Hilbert subspace $\mathcal{A}$ consisting of $\mathcal{N}_{A}$ states $\left\{\left|a_{1}\right\rangle,\left|a_{2}\right\rangle, \ldots\right\}$ whose effective dynamics we wish to describe. This subspace is coupled to another subspace $\mathcal{B}$ consisting of $\mathcal{N}_{B}$ states $\left\{\left|b_{1}\right\rangle,\left|b_{2}\right\rangle, \ldots\right\}$ that we want to adiabatically eliminate. We define the projectors onto the respective subspaces as $P_{\mathcal{A}}$ and $P_{\mathcal{B}}$. The total Hamiltonian of the combined system is given by

$$
H=\left(\begin{array}{cc}
h & V \\
V^{\dagger} & \tilde{H}
\end{array}\right),
$$

where $h \equiv P_{\mathcal{A}} H P_{\mathcal{A}}$ is an $\left(N_{\mathcal{A}} \times N_{\mathcal{A}}\right)$ matrix acting only on $\mathcal{A}, \tilde{H} \equiv P_{\mathcal{B}} H P_{\mathcal{B}}$ is an $\left(N_{\mathcal{B}} \times N_{\mathcal{B}}\right)$ matrix acting only on $\mathcal{B}$, and $V \equiv P_{\mathcal{B}} H P_{\mathcal{A}}$ is an $\left(N_{\mathcal{A}} \times N_{\mathcal{B}}\right)$ matrix coupling both subspaces. Our objective is to obtain an effective Hamiltonian $h^{\text {eff }}$ describing the dynamics within $\mathcal{A}$. The underlying assumption is that the eigenvalues of $h$ are close to the energy $E$, while the eigenvalues of $\tilde{H}$ are detuned from $E$ by values much larger than the elements of $V$, and therefore can be adiabatically eliminated. This is done by writing the eigenvalue problem,

$$
\left(\begin{array}{cc}
h & V \\
V^{\dagger} & \tilde{H}
\end{array}\right)\left(\begin{array}{l}
\phi \\
\chi
\end{array}\right)=E\left(\begin{array}{l}
\phi \\
\chi
\end{array}\right)
$$

where $\phi$ and $\chi$ are column vectors of length $N_{\mathcal{A}}$ and $N_{\mathcal{B}}$, respectively. After matrix multiplication, we obtain the following system of two equations for $\phi$ and $\chi$ :

$$
\begin{aligned}
(E-h) \phi & =V \chi, \\
(E-\tilde{H}) \chi & =V^{\dagger} \phi .
\end{aligned}
$$

By solving Eq. (A3b) and substituting into Eq. (A3a), we obtain

$$
\left(E-H_{\text {eff }}\right) \phi=0,
$$

where $H_{\text {eff }}(E)=h+\delta h$, and

$$
\delta h=V \frac{1}{E-H} V^{\dagger} .
$$

$H_{\text {eff }}$ corresponds to effective Hamiltonians that we have presented in the main text. Notably, this simple expression includes contributions from processes beyond second-order perturbation theory; the order of such processes is encoded in the size of the matrix. In the following appendices, we provide further details on how the effective Hamiltonian was obtained in the three cases studied in the main text.

\section{APPENDIX B: USC EFFECT I: DETAILS FOR TWO PHOTONS EXCITED BY A SINGLE ATOM}

We consider the following two subspaces, with $N_{\mathcal{A}}=2$ and $N_{\mathcal{B}}=12$ :

$$
\begin{aligned}
\mathcal{A}= & \{|n, m,+\rangle,|n+1, m+1,-\rangle\}, \\
\mathcal{B}= & \{|n+1, m, \pm\rangle,|n, m+1, \pm\rangle,|n+2, m+1, \pm\rangle, \\
& |n+1, m+2, \pm\rangle,|n, m-1, \pm\rangle,|n-1, m, \pm\rangle\} .
\end{aligned}
$$

Note that states such as $|n+2, m+1, \pm\rangle$ do not contribute to the effective coupling between the two states in $\mathcal{A}$, but to the Lamb shifts and dispersive cavity-qubit couplings, through processes such as

$|n+1, m+1,-\rangle \rightarrow|n+2, m+1, \pm\rangle \rightarrow|n+1, m+1,-\rangle$.

For simplicity, those processes are neither depicted in Fig. 2 of the main text nor in Figs. 3 and 4. Using $E=\Delta_{1} n+\Delta_{2} m+$ $R$, the correction to the effective Hamiltonian in the subspace $\mathcal{A}$, given by Eq. (A5) has the form

$$
\delta h^{\mathrm{I}}=\left(\begin{array}{cc}
\alpha_{n m}+C & g_{\mathrm{eff}}^{\mathrm{I}} \beta_{n m} \\
g_{\mathrm{eff}}^{\mathrm{I}} \beta_{n m} & -\alpha_{n+1, m+1}+C
\end{array}\right),
$$

where $\alpha_{n m}=\chi_{1} n+\chi_{2} m+\lambda$ and $\beta_{n m}=\sqrt{(n+1)(m+1)}$. This allows us to extract $\chi_{1}, \chi_{2}, \lambda$ and $g_{\text {eff }}^{\mathrm{I}}$, and, omitting any overall shift $C$, to write the effective Hamiltonian $H_{\text {eff }}^{\mathrm{I}}$ in the general form,

$$
\begin{aligned}
H_{\text {eff }}^{\mathrm{I}}= & \Delta_{1} a_{1}^{\dagger} a_{1}+\Delta_{2} a_{2}^{\dagger} a_{2}+(R+\lambda) \tilde{\sigma}_{z} \\
& +\left(\chi_{1} a_{1}^{\dagger} a_{1}+\chi_{2} a_{2}^{\dagger} a_{2}\right) \tilde{\sigma}_{z}+g_{\text {eff }}^{\mathrm{I}}\left(a_{1}^{\dagger} a_{2}^{\dagger} \tilde{\sigma}+\text { H.c. }\right) .
\end{aligned}
$$

The expressions for $g_{\text {eff }}^{\mathrm{I}}, \chi_{1}, \chi_{2}$, and $\lambda$ are provided in Sec. III.

\section{APPENDIX C: USC EFFECT II: DETAILS FOR FREQUENCY CONVERSION}

We consider the following two subspaces, with $N_{\mathcal{A}}=2$ and $N_{\mathcal{B}}=12$ :

$$
\begin{aligned}
\mathcal{A}= & \{|n+1, m,-\rangle,|n, m+1,+\rangle\}, \\
\mathcal{B}= & \{|n+1, m+1, \pm\rangle,|n, m, \pm\rangle,|n+2, m, \pm\rangle, \\
& |n+1, m-1 \pm\rangle,|n, m+2, \pm\rangle,| \pm, n-1, m\rangle\} .
\end{aligned}
$$


Here, the correction that we obtain is

$$
\delta h^{\mathrm{II}}=\left(\begin{array}{cc}
-\alpha_{n+1, m}+C & g_{\mathrm{eff}}^{\mathrm{II}} \beta_{n m} \\
g_{\mathrm{eff}}^{\mathrm{II}} \beta_{n m} & \alpha_{n, m+1}+C
\end{array}\right),
$$

where $\alpha_{n m}$ and $\beta_{n m}$ are defined below Eq. (B1). This allows us to write the general form of the effective Hamiltonian,

$$
\begin{aligned}
H_{\mathrm{eff}}^{\mathrm{II}}= & \Delta_{1} a_{1}^{\dagger} a_{1}+\Delta_{2} a_{2}^{\dagger} a_{2}+(R+\lambda) \tilde{\sigma}_{z} \\
& +\left(\chi_{1} a_{1}^{\dagger} a_{1}+\chi_{2} a_{2}^{\dagger} a_{2}\right) \tilde{\sigma}_{z}+g_{\mathrm{eff}}^{\mathrm{II}}\left(a_{1}^{\dagger} a_{2} \tilde{\sigma}+\text { H.c. }\right) .
\end{aligned}
$$

The expression for $g_{\text {eff }}^{\text {II }}$ is given in Sec. IV. The dispersive coupling rates are in this case given by

$$
\chi_{i}=g^{2}\left(\frac{c^{4}}{2 R+\Delta_{i}}+\frac{s^{4}}{2 R-\Delta_{i}}\right)
$$

and the Lamb shift is

$$
\begin{aligned}
\lambda= & \frac{g^{2}}{2} \frac{c^{4}\left(4 R+\Delta_{1}+\Delta_{2}\right)}{\left(2 R+\Delta_{1}\right)\left(2 R+\Delta_{2}\right)} \\
& +\frac{g^{2}}{2} \frac{s^{4}\left(4 R-\Delta_{1}-\Delta_{2}\right)}{\left(2 R-\Delta_{1}\right)\left(2 R-\Delta_{2}\right)} .
\end{aligned}
$$

The dispersive couplings and the Lamb shift make the diagonal elements of $\delta h^{\mathrm{II}}$ unequal. Since both elements need to be equal in order to achieve complete Rabi oscillations, one needs to introduce a small correction to the resonance condition $\Delta_{1}+\Delta_{2}=2 R$. Introducing the correction $\delta$ such that $\Delta_{1}=2 R f+\delta$ into the final expression of $H_{\text {eff }}$ (which implies the approximation of ignoring $\delta$ during the derivation of $\left.H_{\text {eff }}\right)$ and imposing that the diagonal elements are equal, we are left with the expression for the correction to the general resonance condition:

$$
\delta=(2 n+1) \chi_{1}+(2 m+1) \chi_{2}+2 \lambda .
$$

This expression in terms of $\chi_{i}$ and $\lambda$ coincides with the one obtained for the case of two photons excited by a single atom. Similarly, driving the qubit on resonance does not maximize $g_{\text {eff }}^{\text {II }}$ either. Optimizing the angle gives

$$
\theta^{*}\left(f \lessgtr \frac{1}{2}\right)=\arccos \left[\frac{\sqrt{3+2 f \pm \sqrt{9-4 f+4 f^{2}}}}{2 \sqrt{2}}\right] .
$$

The factor gained with respect to $\theta=\pi / 4$ is also $f$ dependent and has the following expression:

$$
\frac{g_{\mathrm{eff}}^{\mathrm{II}}\left(\theta^{*}\right)}{g_{\mathrm{eff}}^{\mathrm{II}}(\theta=\pi / 4)}=-\frac{\left(-6 f+f^{\prime}+3\right) f^{\prime \prime}}{8 \sqrt{2}(2 f-1)},
$$

where $\quad f^{\prime}=\sqrt{4(f-1) f+9} \quad$ and $\quad f^{\prime \prime}=$ $\sqrt{-2 f\left(2 f+f^{\prime}-2\right)+f^{\prime}+3}$. For the particular case $f=1 / 4$, we find

$$
g_{\text {eff }}^{\mathrm{II}}\left(\theta^{*}\right) \approx 1.76 g_{\text {eff }}^{\mathrm{II}}(\theta=\pi / 4) .
$$

Alternatively, we can compute the optimal detuning $\Delta_{\sigma}$ for a fixed $\Omega$ (instead of fixed $R$ ), which is experimentally more meaningful since varying $\Delta_{\sigma}$ for a fixed $\Omega$ is more straightforward than varying $\theta$ for a fixed $R$ :

$\Delta_{\sigma}^{*}=-\Omega \operatorname{sgn}(1-2 f) \sqrt{-2+\frac{1-\sqrt{1-f(1-f)}|1-2 f|}{f(1-f)}}$.

For the particular case $f=1 / 4, \Delta_{\sigma}^{*} \approx-0.96 \Omega$, which leads to

$$
g_{\text {eff }}^{\mathrm{II}}\left(\Delta_{\sigma}^{*}\right) \approx 1.52 g_{\text {eff }}^{\mathrm{II}}\left(\Delta_{\sigma}=0\right) .
$$

\section{APPENDIX D: USC EFFECT III: DETAILS FOR TWO ATOMS EXCITED BY A SINGLE PHOTON}

We consider the following two subspaces, with $N_{\mathcal{A}}=2$ and $N_{\mathcal{B}}=12$ :

$$
\begin{aligned}
\mathcal{A}= & \{|+,+, n\rangle,|-,-, n+1\rangle\}, \\
\mathcal{B}= & \{|+,-, n+1\rangle,|-,+, n+1\rangle,|+,+, n+1\rangle,|+,-, n\rangle, \\
& |-,+, n\rangle,|-,-, n\rangle,|+,-, n+2\rangle,|-,+, n+2\rangle, \\
& |-,-, n+2\rangle,|-,+, n-1\rangle,|+,-, n-1\rangle, \\
& |+,+, n-1\rangle\} .
\end{aligned}
$$

Here, the correction that we obtain is

$$
\delta h^{\mathrm{III}}=\left(\begin{array}{cc}
2 n \chi+2 \lambda+C & g_{\text {eff }}^{\mathrm{III}} \sqrt{n+1} \\
g_{\text {eff }}^{\mathrm{III}} \sqrt{n+1} & -2(n+1) \chi-2 \lambda+C
\end{array}\right) .
$$

The dispersive coupling and the Lamb shift are given by second-order processes,

$$
\begin{gathered}
\chi=g^{2} \frac{\left(2 R-\Delta_{a}\right) c^{4}+\left(2 R+\Delta_{a}\right) s^{4}}{4 R^{2}-\Delta_{a}^{2}}, \\
\lambda=\chi / 2 .
\end{gathered}
$$

Setting $\Delta_{a}=4 R+\delta$, the optimum resonance condition is given by

$$
\delta=(4 n+2) \chi+4 \lambda=4(n+1) \chi .
$$

For a fixed $R$, we can see that the optimal angle $\theta$ maximizing $g_{\text {eff }}$ is $\theta^{*}=\arctan \left(\sqrt{\frac{1+\sqrt{5}}{3-\sqrt{5}}}\right) \approx 0.356 \pi$, giving the following maximum value of $g_{\text {eff }}^{\text {III }}$ :

$$
g_{\text {eff }}^{\mathrm{III}}\left(\theta^{*}\right)=\sqrt{\frac{11+5 \sqrt{5}}{8}} \frac{g^{3}}{6 R^{2}} \approx 1.67 g_{\mathrm{eff}}^{\mathrm{III}}(\theta=\pi / 4) .
$$

That is, when choosing the optimal angle $\theta^{*}$, we obtain $1.67 \times$ enhancement with respect to the resonant case $\Delta_{\sigma}=0$, which corresponds to $\theta=\pi / 4$. In a similar way, we can express this in terms of the optimal detuning,

$$
\Delta_{\sigma}^{*}=\Omega \sqrt{\frac{14 \sqrt{109}-122}{45}} \approx 0.73 \Omega .
$$

The corresponding value of $g_{\text {eff }}$ is then given by

$$
g_{\mathrm{eff}}^{\mathrm{III}}\left(\Delta_{\sigma}^{*}\right) \approx-1.3 \frac{g^{3}}{6 \Omega^{2}}=1.3 g_{\mathrm{eff}}^{\mathrm{III}}\left(\Delta_{\sigma}=0\right),
$$

giving $1.3 \times$ enhancement with respect to the resonant case, for the same driving amplitude $\Omega$. 
(a)
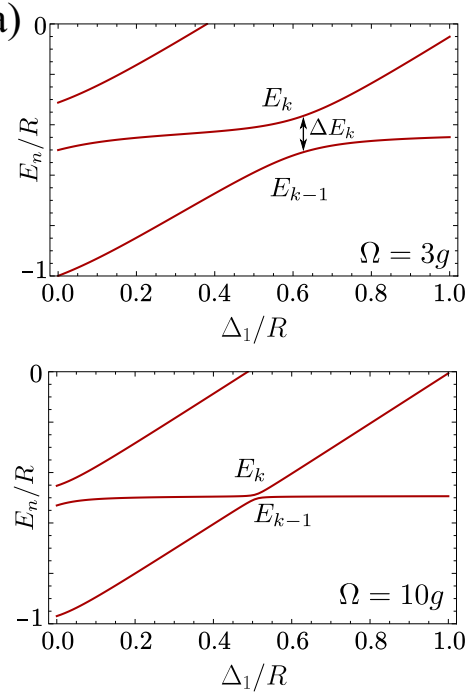

(b)

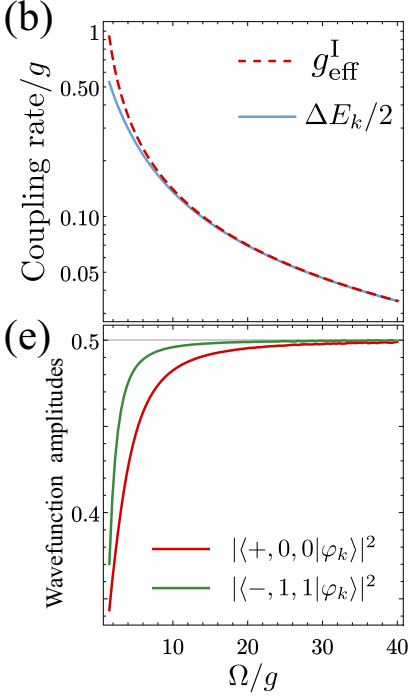

(c)

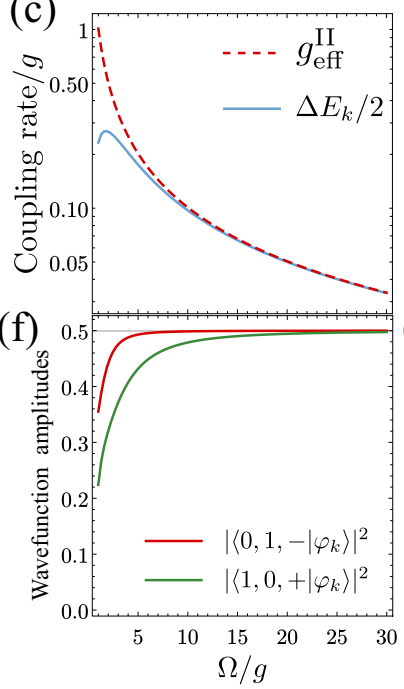

(d)

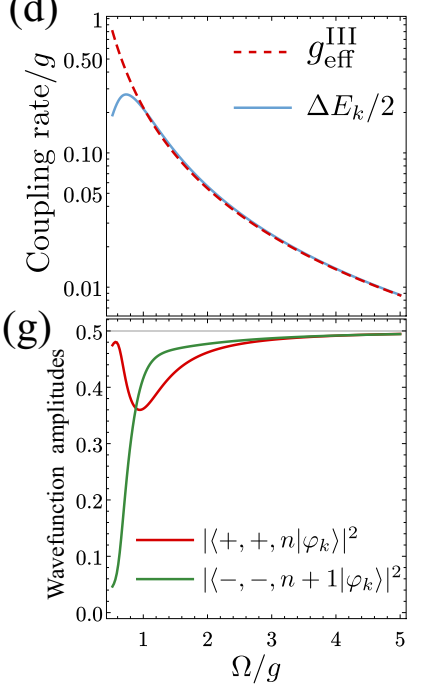

FIG. 7. Validity of the applied perturbation theory for the three examples. (a) Examples of the avoided crossings at two different values of the perturbation parameter $g / \Omega$. Smaller values of $\Omega$ imply larger splitting. (b),(c) Splitting at the avoided crossing (solid curve) vs $\Omega / g$, compared to the effective rates computed from perturbation theory (dashed curve). The perturbation theory works when the two curves overlap. (e),(f) The overlap between the two eigenstates at the avoided crossing and the two states involved in the nonlinear process; when the perturbation theory starts failing, the overlap is reduced, meaning that eigenstates contain contributions from other states.

\section{APPENDIX E: VALIDITY OF THE PERTURBATION THEORY}

In this Appendix, we address the question of the validity of the perturbation theory for the three studied USC effects for large values of the perturbation parameter $g / \Omega$. To do so, we study the energy-level splitting $\Delta E_{k}$ between the two eigenstates $\left|\varphi_{k}\right\rangle$ and $\left|\varphi_{k-1}\right\rangle$ at the avoided-level crossing that we associate to each nonlinear process; see Fig. 7(a). The resulting splitting is compared to the effective coupling rates $g_{\text {eff }}^{\mathrm{I}}, g_{\text {eff }}^{\mathrm{II}}$, and $g_{\text {eff }}^{\mathrm{III}}$ that we have computed from perturbation theory, as we show in Figs. 7(b) and 7(c). In addition, we compute the overlap between the two eigenstates $\left|\varphi_{k / k-1}\right\rangle$ and the two states between which we expect the Rabi oscillations to occur in each of the three cases considered in the text; see Figs. $7(\mathrm{e})-7(\mathrm{~g})$. As $\Omega / g$ is reduced, the effective coupling rates increase. The perturbation theory starts failing at $\Omega / g \lesssim 10$ for cases I and II, and $\Omega / g \lesssim 2$ for case III (which is a third-order process). Below these values of $\Omega / g,\left|\varphi_{k, k-1}\right\rangle$ stop being composed exclusively of the two isolated states that constitute the desired nonlinear process, and the effective coupling rate predicted from perturbation theory departs from the real half splittings between these eigenstates.
[1] S. Haroche, Nobel Lecture: Controlling photons in a box and exploring the quantum to classical boundary, Rev. Mod. Phys. 85, 1083 (2013).

[2] X. Gu, A. F. Kockum, A. Miranowicz, Y.-X. Liu, and F. Nori, Microwave photonics with superconducting quantum circuits, Phys. Rep. 718-719, 1 (2017).

[3] A. F. Kockum and F. Nori, Quantum bits with Josephson junctions, in Fundamentals and Frontiers of the Josephson Effect, Springer Series in Materials Science, Vol. 286, edited by F. Tafuri (Springer, New York, 2019), Chap. 17, pp. 703-741, https://link.springer.com/content/pdf/10.1007\% 2F978-3-030-20726-7_17.pdf.

[4] A. F. Kockum, A. Miranowicz, S. De Liberato, S. Savasta, and F. Nori, Ultrastrong coupling between light and matter, Nat. Rev. Phys. 1, 19 (2019).

[5] P. Forn-Díaz, L. Lamata, E. Rico, J. Kono, and E. Solano, Ultrastrong coupling regimes of light-matter interaction, Rev. Mod. Phys. 91, 025005 (2019).

[6] J. Casanova, G. Romero, I. Lizuain, J. J. García-Ripoll, and E. Solano, Deep Strong Coupling Regime of the JaynesCummings Model, Phys. Rev. Lett. 105, 263603 (2010).
[7] T. Niemczyk, F. Deppe, H. Huebl, E. P. Menzel, F. Hocke, M. J. Schwarz, J. J. Garcia-Ripoll, D. Zueco, T. Hümmer, E. Solano, A. Marx, and R. Gross, Circuit quantum electrodynamics in the ultrastrong-coupling regime, Nat. Phys. 6, 772 (2010)

[8] L. Garziano, R. Stassi, V. Macrì, A. F. Kockum, S. Savasta, and F. Nori, Multiphoton quantum Rabi oscillations in ultrastrong cavity QED, Phys. Rev. A 92, 063830 (2015).

[9] K. K. W. Ma and C. K. Law, Three-photon resonance and adiabatic passage in the large-detuning Rabi model, Phys. Rev. A 92, 023842 (2015).

[10] L. Garziano, V. Macrì, R. Stassi, O. Di Stefano, F. Nori, and S. Savasta, One Photon Can Simultaneously Excite Two or More Atoms, Phys. Rev. Lett. 117, 043601 (2016).

[11] A. F. Kockum, V. Macrì, L. Garziano, S. Savasta, and F. Nori, Frequency conversion in ultrastrong cavity QED, Sci. Rep. 7, 5313 (2017).

[12] A. F. Kockum, A. Miranowicz, V. Macrì, S. Savasta, and F. Nori, Deterministic quantum nonlinear optics with single atoms and virtual photons, Phys. Rev. A 95, 063849 (2017). 
[13] R. Stassi, V. Macrì, A. F. Kockum, O. Di Stefano, A. Miranowicz, S. Savasta, and F. Nori, Quantum nonlinear optics without photons, Phys. Rev. A 96, 023818 (2017).

[14] O. Di Stefano, R. Stassi, L. Garziano, A. F. Kockum, S. Savasta, and F. Nori, Feynman-diagrams approach to the quantum Rabi model for ultrastrong cavity QED: Stimulated emission and reabsorption of virtual particles dressing a physical excitation, New J. Phys. 19, 053010 (2017).

[15] O. Di Stefano, A. F. Kockum, A. Ridolfo, S. Savasta, and F. Nori, Photodetection probability in quantum systems with arbitrarily strong light-matter interaction, Sci. Rep. 8, 17825 (2018).

[16] V. Macrì, F. Nori, and A. F. Kockum, Simple preparation of Bell and Greenberger-Horne-Zeilinger states using ultrastrongcoupling circuit QED, Phys. Rev. A 98, 062327 (2018).

[17] L. Cong, S. Felicetti, J. Casanova, L. Lamata, E. Solano, and I. Arrazola, Selective interactions in the quantum Rabi model, Phys. Rev. A 101, 032350 (2020).

[18] M. Tomka, M. Pletyukhov, and V. Gritsev, Supersymmetry in quantum optics and in spin-orbit coupled systems, Sci. Rep. 5, 13097 (2015).

[19] L. Garziano, R. Stassi, V. Macrì, S. Savasta, and O. Di Stefano, Single-step arbitrary control of mechanical quantum states in ultrastrong optomechanics, Phys. Rev. A 91, 023809 (2015).

[20] J.-M. Pirkkalainen, S. Cho, F. Massel, J. Tuorila, T. Heikkilä, P. Hakonen, and M. Sillanpää, Cavity optomechanics mediated by a quantum two-level system, Nat. Commun. 6, 6981 (2015).

[21] F. Benz, M. K. Schmidt, A. Dreismann, R. Chikkaraddy, Y. Zhang, A. Demetriadou, C. Carnegie, H. Ohadi, B. de Nijs, R. Esteban, J. Aizpurua, and J. J. Baumberg, Single-molecule optomechanics in "picocavities", Science 354, 726 (2016).

[22] V. Macrì, L. Garziano, A. Ridolfo, O. Di Stefano, and S. Savasta, Deterministic synthesis of mechanical NOON states in ultrastrong optomechanics, Phys. Rev. A 94, 013817 (2016).

[23] M. Cirio, K. Debnath, N. Lambert, and F. Nori, Amplified Optomechanical Transduction of Virtual Radiation Pressure, Phys. Rev. Lett. 119, 053601 (2017).

[24] V. Macrì, A. Ridolfo, O. Di Stefano, A. F. Kockum, F. Nori, and S. Savasta, Nonperturbative Dynamical Casimir Effect in Optomechanical Systems: Vacuum Casimir-Rabi Splittings, Phys. Rev. X 8, 011031 (2018).

[25] O. Di Stefano, A. Settineri, V. Macrì, A. Ridolfo, R. Stassi, A. F. Kockum, S. Savasta, and F. Nori, Interaction of Mechanical Oscillators Mediated by the Exchange of Virtual Photon Pairs, Phys. Rev. Lett. 122, 030402 (2019).

[26] A. Settineri, V. Macrì, L. Garziano, O. Di Stefano, F. Nori, and S. Savasta, Conversion of mechanical noise into correlated photon pairs: Dynamical Casimir effect from an incoherent mechanical drive, Phys. Rev. A 100, 022501 (2019).

[27] M. S. Tame, K. R. McEnery, S. K. Özdemir, J. Lee, S. A. Maier, and M. S. Kim, Quantum plasmonics, Nat. Phys. 9, 329 (2013).

[28] F. Todisco, M. De Giorgi, M. Esposito, L. De Marco, A. Zizzari, M. Bianco, L. Dominici, D. Ballarini, V. Arima, G. Gigli, and D. Sanvitto, Ultrastrong plasmon-exciton coupling by dynamic molecular aggregation, ACS Photon. 5, 143 (2018).

[29] B. Munkhbat, M. Wersäll, D. G. Baranov, T. J. Antosiewicz, and T. Shegai, Suppression of photo-oxidation of organic chromophores by strong coupling to plasmonic nanoantennas, Sci. Adv. 4, eaas9552 (2018).
[30] M. A. Sentef, M. Ruggenthaler, and A. Rubio, Cavity quantum-electrodynamical polaritonically enhanced electronphonon coupling and its influence on superconductivity, Sci. Adv. 4, eaau6969 (2018).

[31] F. Schlawin, A. Cavalleri, and D. Jaksch, Cavity-Mediated Electron-Photon Superconductivity, Phys. Rev. Lett. 122, 133602 (2019).

[32] S. Seah, S. Nimmrichter, and V. Scarani, Refrigeration beyond weak internal coupling, Phys. Rev. E 98, 012131 (2018).

[33] J. Galego, F. J. Garcia-Vidal, and J. Feist, Cavity-Induced Modifications of Molecular Structure in the Strong-Coupling Regime, Phys. Rev. X 5, 041022 (2015).

[34] F. Herrera and F. C. Spano, Cavity-Controlled Chemistry in Molecular Ensembles, Phys. Rev. Lett. 116, 238301 (2016).

[35] T. W. Ebbesen, Hybrid light-matter states in a molecular and material science perspective, Acc. Chem. Res. 49, 2403 (2016).

[36] L. A. Martínez-Martínez, R. F. Ribeiro, J. Campos-GonzálezAngulo, and J. Yuen-Zhou, Can ultrastrong coupling change ground-state chemical reactions?, ACS Photon. 5, 167 (2018).

[37] M. Ruggenthaler, N. Tancogne-Dejean, J. Flick, H. Appel, and A. Rubio, From a quantum-electrodynamical light-matter description to novel spectroscopies, Nat. Rev. Chem. 2, 0118 (2018).

[38] P. Nataf and C. Ciuti, Protected Quantum Computation with Multiple Resonators in Ultrastrong Coupling Circuit QED, Phys. Rev. Lett. 107, 190402 (2011).

[39] Y. Wang, J. Zhang, C. Wu, J. Q. You, and G. Romero, Holonomic quantum computation in the ultrastrong-coupling regime of circuit QED, Phys. Rev. A 94, 012328 (2016).

[40] G. Romero, D. Ballester, Y. M. Wang, V. Scarani, and E. Solano, Ultrafast Quantum Gates in Circuit QED, Phys. Rev. Lett. 108, 120501 (2012).

[41] Y. Wang, C. Guo, G.-Q. Zhang, G. Wang, and C. Wu, Ultrafast quantum computation in ultrastrongly coupled circuit QED systems, Sci. Rep. 7, 44251 (2017).

[42] T. H. Kyaw, S. Felicetti, G. Romero, E. Solano, and L.-C. Kwek, Scalable quantum memory in the ultrastrong coupling regime, Sci. Rep. 5, 8621 (2015).

[43] R. Stassi and F. Nori, Long-lasting quantum memories: Extending the coherence time of superconducting artificial atoms in the ultrastrong-coupling regime, Phys. Rev. A 97, 033823 (2018).

[44] I. I. Rabi, On the process of space quantization, Phys. Rev. 49, 324 (1936).

[45] I. I. Rabi, Space quantization in a gyrating magnetic field, Phys. Rev. 51, 652 (1937).

[46] R. H. Dicke, Coherence in spontaneous radiation processes, Phys. Rev. 93, 99 (1954).

[47] J. J. Hopfield, Theory of the contribution of excitons to the complex dielectric constant of crystals, Phys. Rev. 112, 1555 (1958).

[48] E. T. Jaynes and F. W. Cummings, Comparison of quantum and semiclassical radiation theories with application to the beam maser, Proc. IEEE 51, 89 (1963).

[49] M. Tavis and F. W. Cummings, Exact solution for an nmolecule-radiation-field hamiltonian, Phys. Rev. 170, 379 (1968).

[50] K. Le Hur, Kondo resonance of a microwave photon, Phys. Rev. B 85, 140506(R) (2012). 
[51] J. Leppäkangas, J. Braumüller, A. V. Ustinov, M. Weides, and M. Marthaler, Quantum simulation of the spin-boson model in a microwave circuit, Phys. Rev. A 97, 052321 (2018).

[52] M. Goldstein, M. H. Devoret, M. Houzet, and L. I. Glazman, Inelastic Microwave Photon Scattering off a Quantum Impurity in a Josephson-Junction Array, Phys. Rev. Lett. 110, 017002 (2013).

[53] I. Snyman and S. Florens, Robust Josephson-Kondo screening cloud in circuit quantum electrodynamics, Phys. Rev. B 92, 085131 (2015).

[54] A. P. Hines, C. M. Dawson, R. H. McKenzie, and G. J. Milburn, Entanglement and bifurcations in Jahn-Teller models, Phys. Rev. A 70, 022303 (2004).

[55] C. P. Meaney, T. Duty, R. H. McKenzie, and G. J. Milburn, Jahn-Teller instability in dissipative quantum systems, Phys. Rev. A 81, 043805 (2010).

[56] J. Larson, Jahn-Teller systems from a cavity QED perspective, Phys. Rev. A 78, 033833 (2008).

[57] J. Bourassa, J. M. Gambetta, A. A. Abdumalikov, O. Astafiev, Y. Nakamura, and A. Blais, Ultrastrong coupling regime of cavity QED with phase-biased flux qubits, Phys. Rev. A 80, 032109 (2009).

[58] T. Dereli, Y. Gül, P. Forn-Díaz, and Ö. E. Müstecaplioglu, Twofrequency Jahn-Teller systems in circuit QED, Phys. Rev. A 85, 053841 (2012).

[59] L. Garziano, R. Stassi, A. Ridolfo, O. Di Stefano, and S. Savasta, Vacuum-induced symmetry breaking in a superconducting quantum circuit, Phys. Rev. A 90, 043817 (2014).

[60] F. Dimer, B. Estienne, A. S. Parkins, and H. J. Carmichael, Proposed realization of the Dicke-model quantum phase transition in an optical cavity QED system, Phys. Rev. A 75, 013804 (2007).

[61] D. Ballester, G. Romero, J. J. García-Ripoll, F. Deppe, and E. Solano, Quantum Simulation of the Ultrastrong-Coupling Dynamics in Circuit Quantum Electrodynamics, Phys. Rev. X 2, 021007 (2012).

[62] A. L. Grimsmo and S. Parkins, Cavity-QED simulation of qubit-oscillator dynamics in the ultrastrong-coupling regime, Phys. Rev. A 87, 033814 (2013).

[63] J. S. Pedernales, I. Lizuain, S. Felicetti, G. Romero, L. Lamata, and E. Solano, Quantum rabi model with trapped ions, Sci. Rep. 5, 15472 (2015).

[64] S. Felicetti, J. S. Pedernales, I. L. Egusquiza, G. Romero, L. Lamata, D. Braak, and E. Solano, Spectral collapse via twophonon interactions in trapped ions, Phys. Rev. A 92, 033817 (2015).

[65] R. Puebla, M.-J. Hwang, J. Casanova, and M. B. Plenio, Probing the Dynamics of a Superradiant Quantum Phase Transition with a Single Trapped Ion, Phys. Rev. Lett. 118, 073001 (2017).

[66] S. Fedortchenko, S. Felicetti, D. Marković, S. Jezouin, A. Keller, T. Coudreau, B. Huard, and P. Milman, Quantum simulation of ultrastrongly coupled bosonic modes using superconducting circuits, Phys. Rev. A 95, 042313 (2017).

[67] X. Wang, A. Miranowicz, H.-R. Li, and F. Nori, Observing pure effects of counter-rotating terms without ultrastrong coupling: A single photon can simultaneously excite two qubits, Phys. Rev. A 96, 063820 (2017).
[68] I. Aedo and L. Lamata, Analog quantum simulation of generalized Dicke models in trapped ions, Phys. Rev. A 97, 042317 (2018).

[69] W. Qin, A. Miranowicz, P.-B. Li, X.-Y. Lü, J.-Q. You, and F. Nori, Exponentially Enhanced Light-Matter Interaction, Cooperativities, and Steady-State Entanglement Using Parametric Amplification, Phys. Rev. Lett. 120, 093601 (2018).

[70] C. Leroux, L. C. G. Govia, and A. A. Clerk, Enhancing Cavity Quantum Electrodynamics via Antisqueezing: Synthetic Ultrastrong Coupling, Phys. Rev. Lett. 120, 093602 (2018).

[71] N. Lambert, M. Cirio, M. Delbecq, G. Allison, M. Marx, S. Tarucha, and F. Nori, Amplified and tunable transverse and longitudinal spin-photon coupling in hybrid circuit-QED, Phys. Rev. B 97, 125429 (2018).

[72] A. Di Paolo, P. K. Barkoutsos, I. Tavernelli, and A. Blais, Variational quantum simulation of ultrastrong light-matter coupling, arXiv: 1909.08640.

[73] A. Crespi, S. Longhi, and R. Osellame, Photonic Realization of the Quantum Rabi Model, Phys. Rev. Lett. 108, 163601 (2012).

[74] J. Braumüller, M. Marthaler, A. Schneider, A. Stehli, H. Rotzinger, M. Weides, and A. V. Ustinov, Analog quantum simulation of the Rabi model in the ultra-strong coupling regime, Nat. Commun. 8, 779 (2017).

[75] N. K. Langford, R. Sagastizabal, M. Kounalakis, C. Dickel, A. Bruno, F. Luthi, D. J. Thoen, A. Endo, and L. DiCarlo, Experimentally simulating the dynamics of quantum light and matter at deep-strong coupling, Nat. Commun. 8, 1715 (2017).

[76] D. Lv, S. An, Z. Liu, J.-N. Zhang, J. S. Pedernales, L. Lamata, E. Solano, and K. Kim, Quantum Simulation of the Quantum Rabi Model in a Trapped Ion, Phys. Rev. X 8, 021027 (2018).

[77] C. Sánchez Muñoz, E. del Valle, A. González Tudela, K. Müller, S. Lichtmannecker, M. Kaniber, C. Tejedor, J. J. Finley, and F. P. Laussy, Emitters of $N$-photon bundles, Nat. Photon. 8, 550 (2014).

[78] C. Sánchez Muñoz, F. P. Laussy, C. Tejedor, and E. del Valle, Enhanced two-photon emission from a dressed biexciton, New J. Phys. 17, 123021 (2015).

[79] C. Sánchez Muñoz, F. P. Laussy, E. del Valle, C. Tejedor, and A. González-Tudela, Filtering multiphoton emission from state-of-the-art cavity quantum electrodynamics, Optica 5, 14 (2018)

[80] F. Deppe, M. Mariantoni, E. P. Menzel, A. Marx, S. Saito, K. Kakuyanagi, H. Tanaka, T. Meno, K. Semba, H. Takayanagi, E. Solano, and R. Gross, Two-photon probe of the JaynesCummings model and controlled symmetry breaking in circuit QED, Nat. Phys. 4, 686 (2008).

[81] F. Yoshihara, T. Fuse, S. Ashhab, K. Kakuyanagi, S. Saito, and K. Semba, Superconducting qubit-oscillator circuit beyond the ultrastrong-coupling regime, Nat. Phys. 13, 44 (2017).

[82] K. M. Birnbaum, A. Boca, R. Miller, A. D. Boozer, T. E. Northup, and H. J. Kimble, Photon blockade in an optical cavity with one trapped atom, Nature (London) 436, 87 (2005).

[83] H. Häffner, C. F. Roos, and R. Blatt, Quantum computing with trapped ions, Phys. Rep. 469, 155 (2008).

[84] P. Arrangoiz-Arriola, E. A. Wollack, Z. Wang, M. Pechal, W. Jiang, T. P. McKenna, J. D. Witmer, R. Van Laer, and A. H. Safavi-Naeini, Resolving the energy levels of a nanomechanical oscillator, Nature (London) 571, 537 (2019). 
[85] A. Bengtsson, P. Vikstal, C. Warren, M. Svensson, X. Gu, A. F. Kockum, P. Krantz, C. Križan, D. Shiri, I.-M. Svensson, G. Tancredi, G. Johansson, P. Delsing, G. Ferrini, and J. Bylander, Quantum approximate optimization of the exact-cover problem on a superconducting quantum processor, arXiv:1912.10495.

[86] Y. Arakawa, S. Iwamoto, M. Nomura, A. Tandaechanurat, and Y. Ota, Cavity quantum electrodynamics and lasing os- cillation in single quantum dot-photonic crystal nanocavity coupled systems, IEEE J. Sel. Top. Quantum Electron. 18, 1818 (2012).

[87] Y. Chang, A. González-Tudela, C. Sánchez Muñoz, C. Navarrete-Benlloch, and T. Shi, Deterministic Down-Converter and Continuous Photon-Pair Source Within the Bad-Cavity Limit, Phys. Rev. Lett. 117, 203602 (2016). 